\title{
Chemical Bonding Effects in HAADF-STEM Imaging of Light-Element Ceramics
}

\author{
Michael Odlyzko, ${ }^{1}$ Jacob Held, ${ }^{1}$ K. Andre Mkhoyan ${ }^{1}$ \\ ${ }^{1}$ Chemical Engineering and Materials Science, University of Minnesota, Minneapolis, MN 55455
}

Chemical bonding not only determines most of the useful properties of solids; it also alters the electrostatic potentials of bonded atoms, thereby also altering electron scattering from those same atoms. Despite this, it is standard to conduct multislice TEM image simulation [1,2] by modeling the electrostatic potential of a solid as that of a collection of unbonded neutral atoms, which is a computationally convenient approximation known as the "independent atom model" (IAM). IAM simulation has proven especially successful for modeling high-angle annular dark field scanning TEM (HAADF-STEM) imaging [3,4]. However, in two previous studies the authors found that charge redistribution due to chemical bonding can measurably affect HAADF-STEM imaging of polar crystals, because interatomic charge transfer alters probe channeling $[5,6]$.

To test the theoretical prediction of bonding-dependent contrast, we have performed quantitative HAADF-STEM imaging of AIN and MgO single crystals 10-100 $\mathrm{nm}$ in thickness, complemented by bonding-inclusive multislice simulations using a model of atomic potentials that includes bonding contributions, termed the "bonded crystal model" (BCM). Imaging was performed using a FEI Titan G2 60-300 S/TEM operated at $200 \mathrm{kV}$, equipped with a CEOS DCOR probe corrector and a Fischione 3000 HAADF detector (using convergence semi-angles 25-31 mrad, HAADF inner semi-angles 55-68 mrad). The TEMSIM multislice package [7] was used to simulate imaging, with BCM inputs being generated by parameterizing projected atomic potentials calculated using Quantum Espresso [8].

By calibrating the HAADF detector and averaging cross-correlated image frames, high-quality quantitative HAADF-STEM images (Figure 1) were acquired; by carefully measuring thickness, orientation, and effective source size, these images are directly comparable to simulation, albeit without definitive evidence of bonding effects (Figure 2). Results will be discussed in full detail, with emphasis on the delicacy of matching experiment and simulation, either in absolute intensity or in relative contrast. Sensitivity of results to defocus, effective source distribution, and sample orientation will be considered, in addition to the challenges of thickness determination and detector characterization [9].

\section{References}

[1] M.A. O’Keefe et al., Nature 274 (1978), p. 322.

[2] E.J. Kirkland et al., Ultramicroscopy 23 (1987), p. 77.

[3] J.M. LeBeau et al., Phys. Rev. Lett. 100 (2008), p. 206101.

[4] C. Dwyer et al., Appl. Phys. Lett. 100 (2012), p. 191915.

[5] M.L. Odlyzko and K.A. Mkhoyan, Microsc. Microanal. 19 S2 (2013), p. 602.

[6] M.L. Odlyzko and K.A. Mkhoyan, Microsc. Microanal. 20 S3 (2014), p. 154.

[7] E.J. Kirkland, Advanced Computing in Electron Microscopy, (Springer, New York, 2010).

[8] P. Giannozzi et al., J. Phys. Cond. Matter 29 (2009), p. 395502.

[9] This research was supported by NSF DMR-1006706 and NSF MRSEC under award DMR-0819885. Simulations were performed using Minnesota Supercomputing Institute resources. Drs. M. Cococcioni and B. Himmetoglu are thanked for their original density functional theory calculations of bonding charge density in AlN and $\mathrm{MgO}$. 

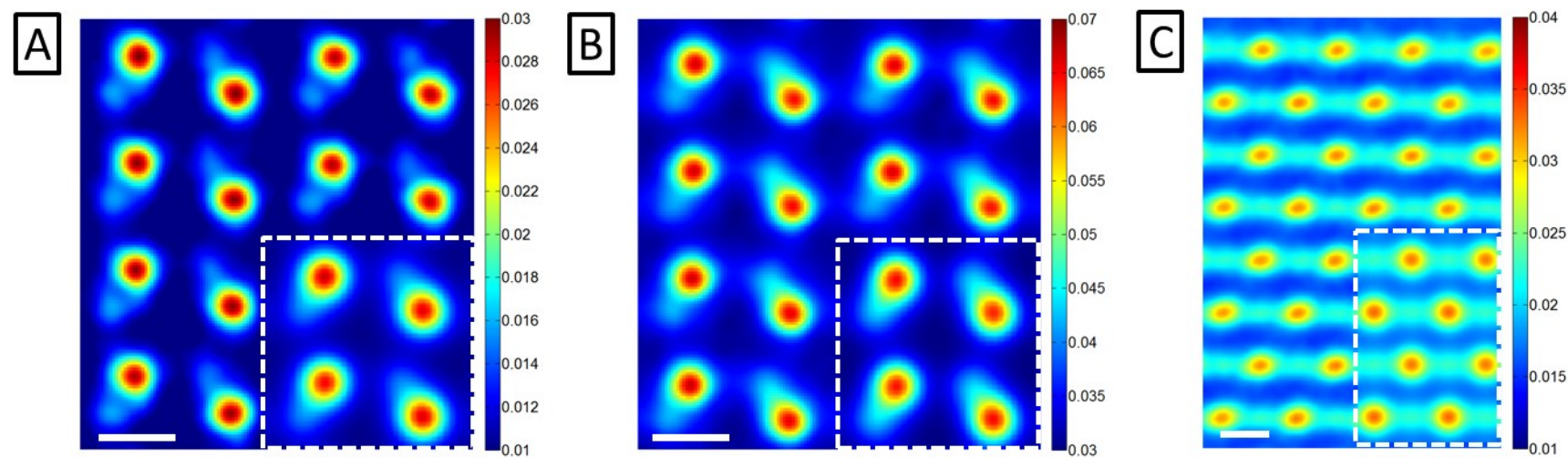

Figure 1. Cross-correlated quantitative HAADF images imaged with a $31 \mathrm{mrad}$ aberration-corrected 200kV probe: (A) $20 \mathrm{~nm}$ thick $<\overline{2} 110>$-oriented AlN imaged with a 55-200 mrad detector, (B) $80 \mathrm{~nm}$ thick $<\overline{2} 110>$-oriented AlN imaged with a $68-200 \mathrm{mrad}$ detector, (C) $40 \mathrm{~nm}$ thick $<110>$-oriented $\mathrm{MgO}$ imaged with a 68-200 mrad detector. Inset BCM simulations illustrate the close agreement between experiment and simulation. HAADF intensity is normalized to incident beam current and each scale bar is $0.2 \mathrm{~nm}$ in length.

A

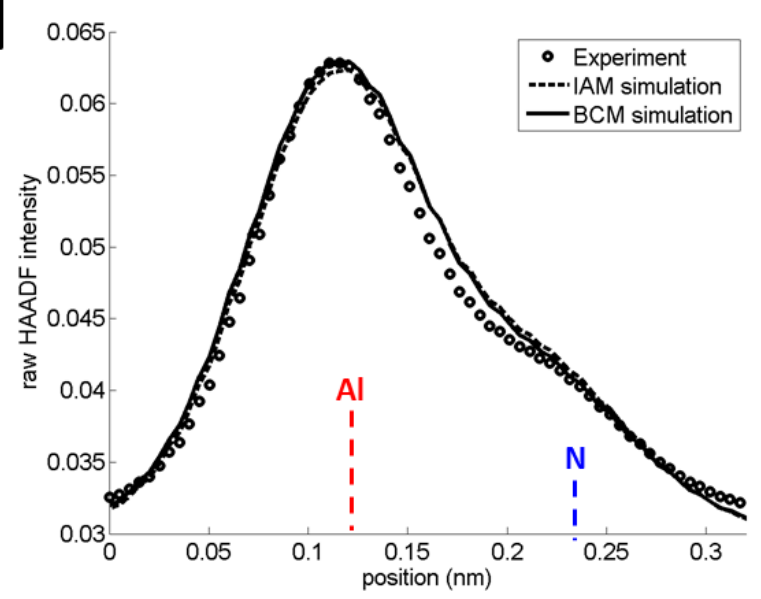

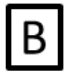

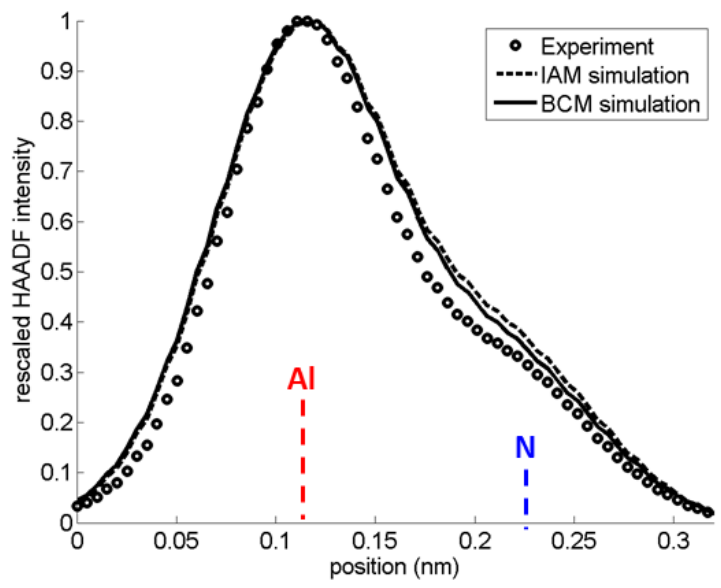

Figure 2. Quantitative linescan analysis of simulated vs. experimental data, same case as $1 \mathrm{~B}$ above $(80$ nm thick $<\overline{2} 110>$-oriented AlN, $200 \mathrm{kV}, 31 \mathrm{mrad}$ probe, 68-200 mrad detector, gaussian defocus): (A) raw data, (B) background-subtracted data normalized to maximum. In this case, differences between experimental data and either simulation are greater than the differences between the two different simulation types, showing that any bonding effect is overshadowed by other factors (particularly effective source-size determination) even in the case of a sound experiment-simulation match. 